\title{
La empresa y los accidentes en el ámbito laboral. Caso de algunas empresas peruanas
}

\section{The company and accidents in the workplace. Case of some Peruvian companies}

Carlos Eduardo Armas Morales

\begin{abstract}
Resumen
La Seguridad y Salud en el Trabajo en el Perú: Ley No. 29786, promulgada en el 2011, se contradice con la importancia de la seguridad del trabajador en el trabajo, asumiendo que es quien genera el ingreso de las familias y asimismo es contribuyente tanto de la renta empresarial como, a nivel macro, del Producto Bruto Interno. Desde esa óptica, las empresas deben comportarse con liderazgo e idoneidad, asumiendo el cumplimiento de la ley e implementado ambientes laborales que prevengan y protejan de riesgos de accidentes en áreas laborales, con el fin de evitarlos o minimizarlos. En esta perspectiva, el artículo explora y describe bajo un enfoque cualitativo, si un grupo de empresas tiene un comportamiento idóneo en materia de seguridad en el trabajo. Los resultados obtenidos indican que los grupos de empresas evaluadas no asumen un comportamiento adecuado para tal finalidad.
\end{abstract}

\section{Palabras clave}

Empresa, accidentes de trabajo, derechos a la seguridad en el trabajo.

\section{Códigos de clasificación JEL: J28, D21, I31}

\begin{abstract}
Occupational Health and Safety in Peru: Law No. 29786, promulgated in 2011, is consistent with the importance of worker safety at work, assuming that it is who generates the income of families and is also a contributor to his work both in business income and at the macro level of the Gross Domestic Product. From this point of view, companies must behave with leadership and suitability assuming compliance with the law implemented work environments that prevent and protect against accidents in occupational areas in order to avoid or minimize them. In this perspective, the article explores and describes under a qualitative approach, if a group of companies have an ideal behavior in terms of occupational safety. The results obtained indicate that the groups of companies evaluated do not assume adequate behavior for this purpose.
\end{abstract}

\section{Keywords}

Company, work accidents, rights to safety at work.

1 Universidad Nacional Mayor de San Marcos UNMSM. Correo electrónico: carmasm@unsm.edu.pe Código ORCID: 0000-0002-4302-565X. 


\section{Introducción}

La seguridad y salud en el trabajo en cualquier empresa debe basarse en el respeto a la vida y el bienestar del trabajador; pues si bien no existe en Perú una norma específica constitucional que taxativamente prescriba a la seguridad y salud en el trabajo como derecho humano; si está consagrado el artículo 2 numeral 1: "el derecho a la vida...integridad moral, psíquica y física y a su libre desarrollo y bienestar"; es evidente que este tratamiento jurídico constitucional aborda la seguridad y la salud en el trabajo, que por demás, concurre a la par con normas internacionales (Perú ha suscrito y ratificado diversas normas en esta materia) plasmadas en la política y legislación de la especialidad.

En el caso peruano, la ley de Seguridad y Salud en el Trabajo, Ley No. 29786 (en adelante Ley de SST), inaugura por primera vez la regulación de esta materia con rango de ley, sin embargo, ello no quiere decir, que no hubo antes regulación; sí la hubo, pero con normas de menor jerarquía (Reglamento de Seguridad en el Trabajo: D.S. No. 009-2005-TR). Con dicha norma legal, más la política respectiva, se trata de prevenir y proteger los riesgos a los trabajadores, que ejercitan su trabajo para sostener a sus familias y de la misma manera para que la empresa produzca bienes y servicios que demanda la sociedad sin contratiempos, amparados en que "la política legislativa y reglamentaria puede ayudar a la reducción de lesiones y muertes, considerando como un punto relevante la capacitación" (Tagle, y del Carmen, 2019) e igualmente, su difusión permanente para el conocimiento de todos los trabajadores y directivos; en consecuencia, un accidente de trabajo, sea fatal o no, impacta y afecta tanto al trabajador como a las empresas lo que permite valorar el principio: que a mayor seguridad menor número de accidentes, y desde luego mayor sanidad del trabajador, más estabilidad económica de la empresa; y desde ámbitos macroeconómicos, menos gastos del ente estatal por pérdidas de vida o discapacidades de los trabajadores.

Ello se fundamenta en diversos planteamientos teóricos que hacen referencia a "Los costes y el impacto económico de las consecuencias de la prevención se generan en principio en el ámbito empresarial, por tanto, el análisis del comportamiento empresarial a este respecto es clave para el análisis económico de la prevención" (Olavarri, 2014, p. 161) y desde otro ángulo, el trabajador es un ser humano que tiene el derecho de trabajar en condiciones adecuadas en ámbitos laborales, a fin de evitar riesgos que puedan impactar su vida e integridad física, y desde luego, se reitera que, todo accidente laboral es una pérdida económica para la empresa.

De otro lado, según la conclusión prescrita por Ortega, Rodríguez y Hernández (2017):

Es evidente que la normatividad nacional vigente no es efectiva en la protección integral de la fuerza laboral, puesto que no hay manera de verificar a ciencia cierta si todas las empresas de la economía formal están cumpliendo o no con 
dotar a sus empleados con los EPP necesarios, y en la calidad que se requieren. Mucho menos, puede evidenciarse tal situación en la economía informal ( $\mathrm{p}$. 173).

En tal sentido el interés de este artículo fue explorar el comportamiento empresarial (grupo de empresas peruanas), en el entendido que, si el empresario peruano (periodo de estudio entre los años 2014 al 2018) es consciente de las implicancias de los accidentes de trabajadores en sede laboral, refiriendo que estos se traducen en horas no trabajadas, pérdidas materiales de equipos, de infraestructura, etc., así como la disminución de la productividad y rentabilidad empresarial; en consecuencia, si a la gerencia empresarial se le encomienda como objetivo maximizar las ganancias, esta debe optar por organizar en forma eficiente, entre otros temas, la prevención de riesgos de accidentes. Aspectos que el grupo de empresas evaluadas en este estudio no lo realiza adecuadamente, conforme se observa en los resultados.

Visto así, y siguiendo la línea obligacional del empresario (artículo 48 de la Ley SST), de ser el líder en materia de seguridad laboral, se permite que la prevención de accidentes, asumiendo el buen comportamiento del empresario a favor de la seguridad en el trabajo, es un objetivo fundamental de la gerencia empresarial, dado que es estratégico que las gerencias de seguridad y salud en el trabajo formen parte de la estrategia general de la empresa.

\section{Marco teórico}

Antes de abordar estudios previos conexos y la revisión bibliográfica, se exponen algunos conceptos, que definen los vocablos: accidente e incidente; fundamentales para el desarrollo del artículo. Recurriendo a la Decisión No. 851 del GRAN, instrumento andino del cual Perú es signatario. En una de sus disposiciones generales dado por el artículo 1, literal $\mathrm{n}$ ), define como accidente de trabajo:

a todo suceso repentino que sobrevenga por causa o con ocasión del trabajo, y que produzca en el trabajador una lesión orgánica, una perturbación funcional, una invalidez o la muerte. Mas el siguiente párrafo expresa: Es también accidente de trabajo aquel que se produce durante la ejecución de órdenes del empleador, o durante la ejecución de una labor bajo su autoridad, aun fuera del lugar y horas de trabajo.

Es decir, el trabajo ceñido al escenario físico fijado por el empleador dentro de la empresa es restringido, ya que el trabajador por razones del trabajo o la naturaleza del puesto laboral, puede discurrir en escenarios fuera del entorno habitual, más aún, si esto es por órdenes de su empleador, sin embargo agrega la norma: "Las legislaciones de cada país podrán definir lo que se considere accidente de trabajo respecto al que se produzca durante el traslado de los trabajadores desde su residencia a los lugares de trabajo o viceversa". 
El reglamento de la ley peruana de SST (Decreto Supremo No. 005-2012-TR) indica que accidente de trabajo: "Es todo suceso repentino que sobrevenga por causa o con ocasión al trabajo y que produzca en el trabajador una lesión orgánica, una perturbación funcional, una invalidez o la muerte". Siendo así el accidente puede producirse en itinerario de ida o regreso al puesto de trabajo o centro laboral cuando haya sido por una orden del empleador y que es concordante con el artículo 54 de la Ley SST (deber de prevención del empleador) que extiende su aplicación al periodo en el cual el trabajador se desplaza hacia el centro de trabajo, siendo más, cuando este se produzca fuera de las horas y jornada de trabajo. Toda esta gama de situaciones se contempla en las normas sectoriales o analizando la naturaleza de la actividad (teletrabajo, coworking), la condición de trabajo o también cuando el desplazamiento se realice en un medio de transporte brindado por el empleador, sea esta en forma directa o a través de terceros (artículo 54 de la Ley SST y 91 del Reglamento). Este último aspecto resulta peculiarmente importante, pues, a la fecha, los accidentes producidos durante el traslado de los trabajadores hacia el centro de trabajo no se encuentran cubiertos por el Seguro Complementario de Trabajo de Riesgo.

En cuanto a incidente, sin ser más dañoso que un accidente, es inevitable conocer, dado su conexidad con un accidente, pues son el preludio de un accidente si no se atiende o se le da la importancia debida. En ese sentido Raffo sintetiza en dos notas la diferencia. "Nota 1: un accidente es un incidente que da lugar a lesión, enfermedad o victima", "Nota 2: un incidente en el que no hay una lesión, enfermedad ni víctima mortal también se puede denominar como 'casi accidente’ (Raffo, 2016, p. 61).

Ahora bien, en la actualidad se vive en una "sociedad de riesgo" (Silva, 2001), debido a las transformaciones tecnológicas, sociales y culturales que impactan en todos los campos de la vida humana; correspondiendo a los seres humanos, no solo por instinto, si no por racionalidad, el resguardo de la propia vida, la integridad física y mental, ante la amenaza de riesgos que le causarían daño o perjuicio. Más aún, como dice Feo, "existe un nuevo patrón tecnológico que ha generado una recomposición productiva con nuevas formas de organización laboral: trabajo flexible, teletrabajo, maquila, tercerización..." (2010, p. 92). Agregando que, "hay una combinación y acumulación de riesgos, seguimos enfrentando los viejos: físicos, químicos, biológicos; al tiempo que lidiamos con los riesgos de las nuevas formas de organización del trabajo, robotización y flexibilización" (2010, p. 92); que, si no se adecúan las condiciones laborales a esta realidad podría haber un posible incremento de accidentes laborales. En tal sentido resulta necesario la vigilancia de las condiciones de trabajo visionado en los interrogantes: ¿Qué está cambiando? ¿Cuáles serán las consecuencias para la salud de los trabajadores? ¿Qué medidas deben tomarse para prevenir posibles consecuencias perjudiciales y minimizar los riesgos? (Pinilla, 2019).

Palacios y otros (2014) refieren, usando el concepto amplio de trabajo, que está organizado bajo una sociedad capitalista con base lógica a la ganancia empresarial, puede explicar tanto las formas de producir y distribuir los bienes como las formas de 
exposición a factores de riesgo y a las condiciones en las que el trabajador realiza su labor en su puesto de trabajo, concluyendo que “...los requerimientos del proceso de trabajo capitalista organizan toda la vida social y, por tanto, las formas de enfermar y morir de la población" (2014, pp. 172-173).

Agudizando la mirada, si la empresa es la que organiza los recursos humanos para la producción de un bien o servicio debe tener como regla observar el ambiente laboral que ocupa el trabajador, inclusive, en el sentido de estar prevenidos para los riesgos de accidentes, incluso enfermedades, pues lo contrario sería atentatorio, no solo contra el trabajador, si no contra la misma empresa. Al respecto Riaño y Palencia (2016) resumen lo dicho, indicando que un objetivo básico de la seguridad y la salud es proteger a los trabajadores de los peligros en el lugar de trabajo que pueden generar, muerte, enfermedad e invalidez, con el fin de crear y mantener un ambiente seguro y saludable, y para que contribuyan a aumentar la calidad, productividad y competitividad empresarial. En tal sentido, la empresa debe, no por una necesidad filantrópica, sino porque quien colabora para la rentabilidad empresarial tiene que estar en ópticas condiciones de trabajo, más, como lo expresa Porter: "que las empresas con éxito necesitan una sociedad sana..." (2009, p. 559).

De otro lado Chamochumbi, refiriéndose al caso peruano expresa que: "la empresa privada ha mejorado sus métodos y proceso industriales, aunque su trabajo informal todavía es un lastre y una gran parte de accidentes se da en estos sectores" (2014, p. 13). Acotando en general que el empresariado tiene un mejor comportamiento en relación con años anteriores, empero deslinda que, el problema de seguridad sea solo del empresariado, pues alega que es un problema de educación y conciencia que se inicia en el colegio, aunque se diría con mayor amplitud que esto debe iniciarse desde el hogar.

Desde otro ángulo se discurrió por un grupo de investigaciones plasmadas en artículos de investigación y tesis sobre seguridad y salud en el trabajo (en adelante SST) buscando enfoques que rescaten el sentido del comportamiento empresarial en materia de SST.

Así, Céspedes y Martínez sobre la SST en el sistema empresarial cubano, expresan que "En ocasiones, los sistemas de gestión de seguridad y salud en el trabajo implementado en nuestras empresas, no cumplen con su objetivo fundamental, como es disminuir o evitar la ocurrencia de incidentes, o accidentes de trabajo..." (2016, p. 2), denotando incumplimiento del deber de prevención y un comportamiento poco consecuente con la responsabilidad social empresarial (en adelante RSE), atinente al trabajador; es decir, un comportamiento inadecuado a favor de la SST. En la misma línea de la responsabilidad social se inserta el estudio, realizado por Figueroa, Ribet, Garrido, Ramos \& Enrique (2013), que enlaza la gestión de riesgos laborales en las empresas con la responsabilidad social, arguyendo que ante la diversidad de riesgos laborales a los que se enfrentan las empresas y la necesidad de tomar conciencia de su gestión, resulta indiscutible la enorme importancia de estos riesgos en el entendi- 
do de evitar accidentes de trabajo como parte de la responsabilidad de la empresa y, desde luego, ligado a un comportamiento de resguardar la SST.

En esa misma línea dual de trabajo (RSE y SST), Suasnavas y otros estudian el panorama actual de las empresas ecuatorianas, concluyendo que la RSE es un tema de interés actual en Latinoamérica, no obstante, en Ecuador las empresas asocian la responsabilidad con acciones y compromisos con el medio ambiente, el entorno comunitario, con programas de ayuda a damnificados, sin considerar la seguridad y salud del trabajador; este hecho posiblemente es debido al desconocimiento de la gerencia (Suasnavas, Andrade, Dávalos y Cárdenas, 2019), lo que denota el poco compromiso, y desde luego, un débil comportamiento empresarial en materia de SST por el empresariado ecuatoriano que podemos trasladarlo a Latinoamérica.

Asimismo, la tesis de Hernández indica enfáticamente que:

A los dueños de las compañías les debería interesar ser responsables con sus trabajadores porque son ellos quienes con su entrega diaria producen los bienes y servicios que posibilitan la existencia de la organización. Ser socialmente responsables con los trabajadores es una cuestión de ética, pero también evita costos indeseados de rotación, accidentes o enfermedades, e incluso costos asociados (2014, pp. 13-14).

Participa en el interés de un comportamiento responsable en salud y seguridad en el trabajo como premisa básica de la responsabilidad social de la empresa.

Una tesis doctoral española sobre análisis y evaluación de la investigación de accidentes laborales como técnica preventiva, denota la unidad de la RSE y SST, concierne a la investigación de accidentes como una técnica analítica para conocer las causas de ocurrencia de accidentes debido a las falencias en los sistemas de SST, y que apunta a proponer un modelo común para Europa (Salguero, 2017), que sugiere desde otra mirada, la concurrencia y evaluación de factores cuantitativos y cualitativos del comportamiento empresarial en esta materia.

Ahora bien, analizando aspectos más prácticos concernientes a la realidad de la seguridad y salud en el trabajo en Perú, en esta segunda década del siglo actual, el trabajo de Ugaz y Soltau (2011) utiliza las notificaciones del Ministerio de Trabajo y Promoción Social y manifiesta que pese a los esfuerzos existentes por optimizar la defensa de los trabajadores en tales materias (seguridad y salud en el trabajo), el número de accidentes sigue siendo preocupante a nivel nacional, ya que los accidentes (no mortales) y enfermedades siguen incrementándose; así, entre septiembre del 2010 y septiembre del 2011, ascendieron a 3.307 y el de las enfermedades a 53.

Mejía, Cárdenas y Gomero (2015) en un artículo sobre accidentes y enfermedades laborales en Perú, de acuerdo con las notificaciones del MTPE identificaron en el año 2012, 15.508 accidentes no mortales, 19.412 para 2013 y 14.750 en el año 2014; se vislumbra que el número de accidentados de este tipo siguen incrementándose en los años siguientes (Tabla 1), notándose un despropósito en cuanto al comportamiento 
empresarial sobre la SST, que desde luego será apreciado y solventado más adelante con las evaluaciones realizadas.

Tabla 1. Accidentes no mortales en el periodo 2014-2018

\begin{tabular}{cccccc}
\hline & Accidentes & No & Mortales & & \\
\hline Año & 2014 & 2015 & 2016 & 2017 & 2018 \\
\hline No. Eventos & 14.750 & 20.938 & 20.913 & 15.665 & 20.479 \\
\hline
\end{tabular}

Fuente: Elaboración propia (datos de MTPE).

En la Universidad Nacional del Centro del Perú, Ruiz (2017), en su tesis Diseño e implementación del sistema de gestión en seguridad y salud ocupacional en la planta de Yauris, señala que el principal activo de cualquier institución es el personal que labora. Se pueden presentar accidentes que pueden incapacitar a los miembros de la institución, lo que ocasionaría no solo pérdidas del factor humano, sino que también la institución tendría que incurrir en altos costos, lo cual involucra pérdidas en la dualidad empresa-trabajador; enfatizando la importancia de la SST y sugiriendo un buen comportamiento a todos los actores, que son todos los que laboran, y que de acuerdo con la ley, es el empresario quien lidera esta función.

Como se induce en estos últimos estudios, el empresariado denota preocupación en mejorar la prevención y el resguardo del trabajador en espacios laborales, de ahí la pertinencia del presente trabajo, partiendo del fundamento de que la prevención o el actuar contra los riesgos laborales en el trabajo -eliminando o disminuyéndolos- implica la protección al trabajador que desarrolla sus habilidades manuales y cognitivas, para satisfacer las necesidades básicas de su familia, siendo igualmente un compromiso de la dirección empresarial de resguardar la renta empresarial.

\section{Metodología}

La investigación aborda un enfoque cualitativo, utiliza el modelo conceptual inductivo y conjuga un diseño fenomenológico que pretende explorar, describir y entender el fenómeno de seguridad y accidentalidad en el trabajo desde el punto de vista de las empresas o grupo de empresas que está integrado por personas (propietarios, gerentes, directores y trabajadores), cuyas experiencias se ven reflejadas en el comportamiento empresarial, referidos a la prevención de la seguridad y salud en el trabajo durante el periodo del 2014 al 2018.

Se evaluaron grupos de empresas heterogéneas y de diversos tamaños, se incluyeron 16 empresas, y se usaron diversas técnicas: la observación para establecimientos comerciales o de servicios, referido a las galerías que agrupan empresas unipersonales, microempresas y negocios, la técnica documental (sobre cuatro grandes empresas con sede en Lima, aunque los accidentes concurrieron en algunos casos 
en provincias) usando casaciones judiciales; asimismo, siete empresas de diversos sectores reportados en las publicaciones de diarios en el periodo evaluado y sobre accidentes laborales ocurridos en Lima o provincias.

Con la técnica de la encuesta se evaluaron cuatro empresas más: en primera instancia, tanto a trabajadores como a los encargados de la seguridad y salud de las empresas, a estas últimas se les tuvo que excluir, pues todas afirmaban las bondades de su gestión, por lo que se consideró una información dudosa, supliéndolo con entrevistas de expertos para cubrir este aspecto del comportamiento empresarial en materia de SST. Se validaron encuestas de 30 trabajadores de estas cuatro empresas.

Si bien los grupos empresariales evaluados corresponden, en su mayoría, a Lima (la sede puede ser Lima, pero la ocurrencia del evento accidente e incidente en algunos casos es en provincias). De otro lado, la capital peruana concentró el $48.1 \%$ del PBI en el 2017 (Agencia Andina de Noticias, 2019) y por tanto, el gran porcentaje de empresas y trabajadores residen en la capital, no obstante, es aplicable a cualquier lugar del país, debido a que se tiene una misma ley y cultura nacional, así como una misma estadística nacional. Por esto, se tuvo como base la data publicada en la página Web del Ministerio de Trabajo y Promoción de la Empresa del Perú (MTPE) que incluye las estadísticas de accidentes mortales y no mortales e incidentes de diversas empresas a nivel nacional.

\section{Resultados}

\section{Estadísticas}

A partir de estadísticas del MTPE (periodo 2014-2018) se ha tabulado en tablas: Accidentes no mortales (tabla 1), Mortales (tabla 2) e Incidentes (tabla 3) y tasas para ser evaluados de acuerdo con nuestro objetivo y que se muestran integrados en las figuras 1 y 2 . La tasa de accidentes mortales o no e incidentes se han calculado teniendo como referencia el número de eventos sobre el número de trabajadores de población económicamente activa (PEA) peruana por un millón de habitantes.

El nivel de accidentes no mortales (tabla 1) alcanzó en ese periodo máximo 20.938 (2015) y un mínimo 14.750 (2014), la que comparado con los 3.307 que reporta Ugaz y Soltau (2011) entre septiembre del 2010 y septiembre del 2011 (13 meses) es incipiente e inclusive con los de los años 2012, 2013 mostrados por Mejía et. al (2015); siendo dichas cifras, obtenidas de la data o notificaciones del MTPE, notándose que desde el 2012 ya se aplicaba la ley y el reglamento, en consecuencia se presume que este incremento abrupto se podría deber a la obligatoriedad de notificar por la norma legal actual, ya que anteriormente las empresas eran reticentes para reportar los accidentes laborales. 
De otro lado, al 2018 los accidentes no mortales notificados según el MTPE superaban aproximadamente en un 25\% a los del 2017 (tabla 1), lo cual indica pocos cambios en el comportamiento empresarial en materia de seguridad en el trabajo. Comparando estas cifras, con datos sobre estadísticas de accidentes no mortales en el total de sectores de España y Francia, la tendencia es a reducirlos, así, en más de dos décadas, España de 6846 en 1990 lo redujo a 2839 en 2012 y Suecia de 1050 en 1993 a 662 en 2012 (Universidad de Valencia, 2016).

Los accidentes mortales (tabla 2) en promedio desde el 2014 se mantienen con escasa variación anual, lo que se refleja en la tasa de mortalidad anual, que indicaría que no hay una actitud de mejora, sin embargo, en el Congreso de Prevención de Riesgos Laborales organizado por La Positiva (una empresa de seguros) en el año 2016 , indica que esta tasa (inferida para el 2015) estaba entre $13.5 \%$ y $13.8 \%$, siendo considerado como nivel crítico, sin embargo, el cálculo obtenido con los datos que se maneja en esta investigación para ese año es de 10.4 (tabla 2), no obstante, la diferencia con Colombia, que para el mismo año presentó una tasa de $6.9 \%$, es decir menor. Siendo que los países como EE.UU., Europa y Japón tienen una tasa de fatalidad que bordea el 3.5\% (Gestión, 2016). Esta tasa es un indicador en el que el empresariado peruano tiene mucho que mejorar, se podría decir que cuantitativamente se han incrementado las empresas por eso se tiene esta tasa sin variación significativa, pero también cada vez existen posibilidades tecnológicas de mejoras, tanto en procedimientos como en equipos.

Tabla 2. Accidentes mortales en el periodo 2014-2015

\begin{tabular}{cccccc}
\hline & Accidentes & & \multicolumn{2}{c}{ Mortales } & \multicolumn{2}{c}{ (Tasa de mortalidad) } \\
\hline Año & 2014 & 2015 & 2016 & 2017 & 2018 \\
\hline No. Eventos & $128(7.46)$ & $179(10.40)$ & $150(8.64)$ & $162(8.89)$ & $151(8.24)$ \\
\hline
\end{tabular}

Fuente: Elaboración propia (datos de MTPE).

El número de incidentes (tabla 3), que son accidentes sin daño o escaso daño, sin embargo, podrían convertirse en accidentes si no se toman las providencias del caso. En los últimos años (Número de eventos y tasa de incidentes anuales) indican que existe una tendencia a la baja desde el 2014, lo que mostraría, por lo menos en este rubro, una cierta preocupación del empresariado, desde luego que estudios cuantitativos específicos y exhaustivos serían más esclarecedores.

Tabla 3. Incidentes en los últimos cinco años

\begin{tabular}{cccccc}
\hline & \multicolumn{3}{c}{ Incidentes } & \multicolumn{3}{c}{ (Tasa de incidentes) } \\
\hline Año & 2014 & 2015 & 2016 & 2017 & 2018 \\
\hline No. Eventos & $870(50.67)$ & $862(50.09)$ & $726(41.83)$ & $615(34.18)$ & $501(27.33)$ \\
\hline
\end{tabular}

Fuente: Elaboración propia (datos de MTPE). 
Las figuras 1 y 2 muestran las tendencias de accidentes e incidentes, habiendo cierta disminución de los incidentes tanto en eventos y tasas que pueden mejorar resultados en el futuro si sigue la tendencia, pero en cambio no hay una variación importante en los accidentes (no mortales y mortales) para unirse y mejorar el comportamiento empresarial.

Figura 1. Accidentes e incidentes en el periodo 2011-2014 en el Perú

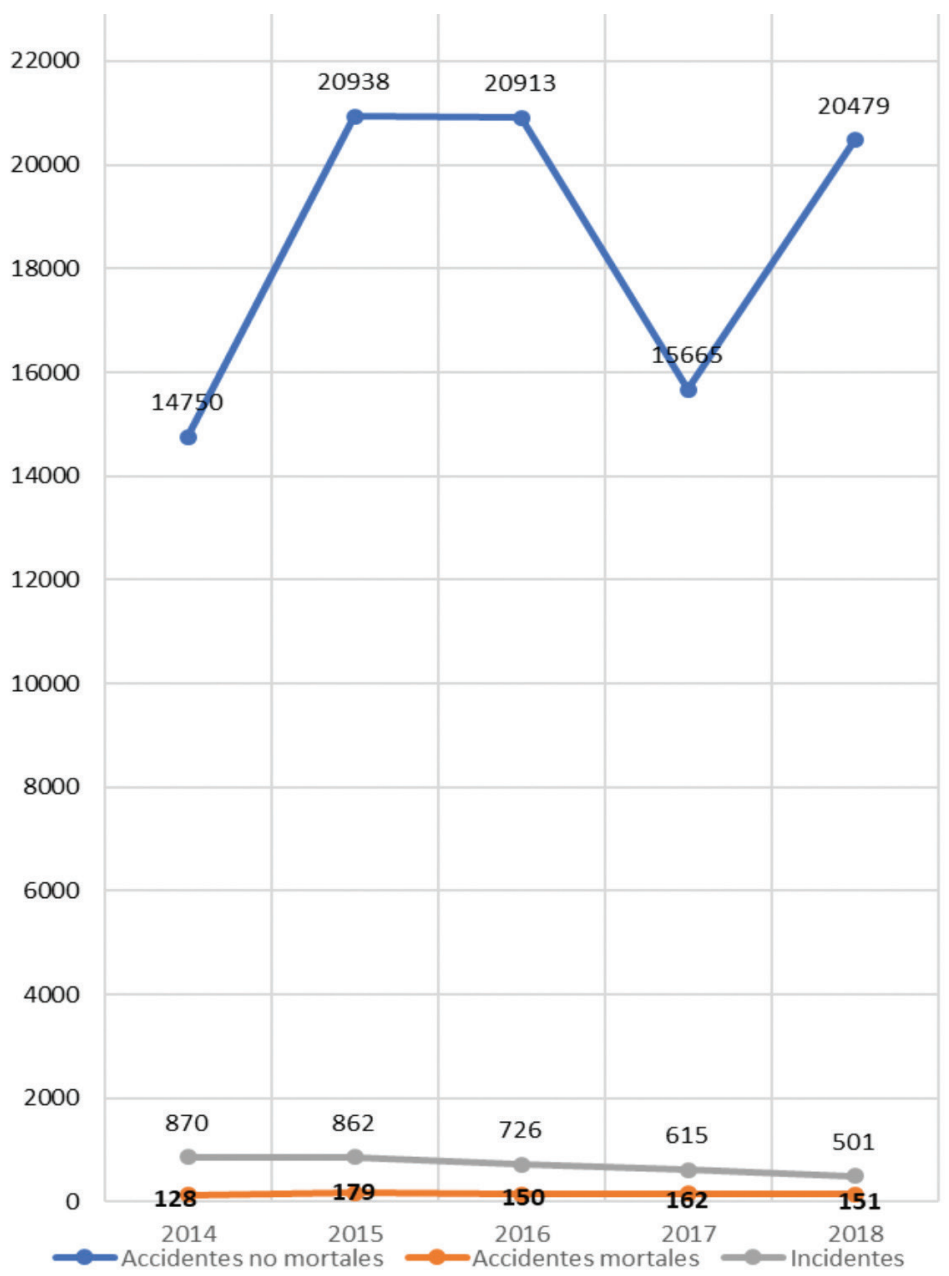

Fuente: Elaboración propia. 
Figura 2. Comparación de tasa de accidentes mortales, incidentes en el Perú

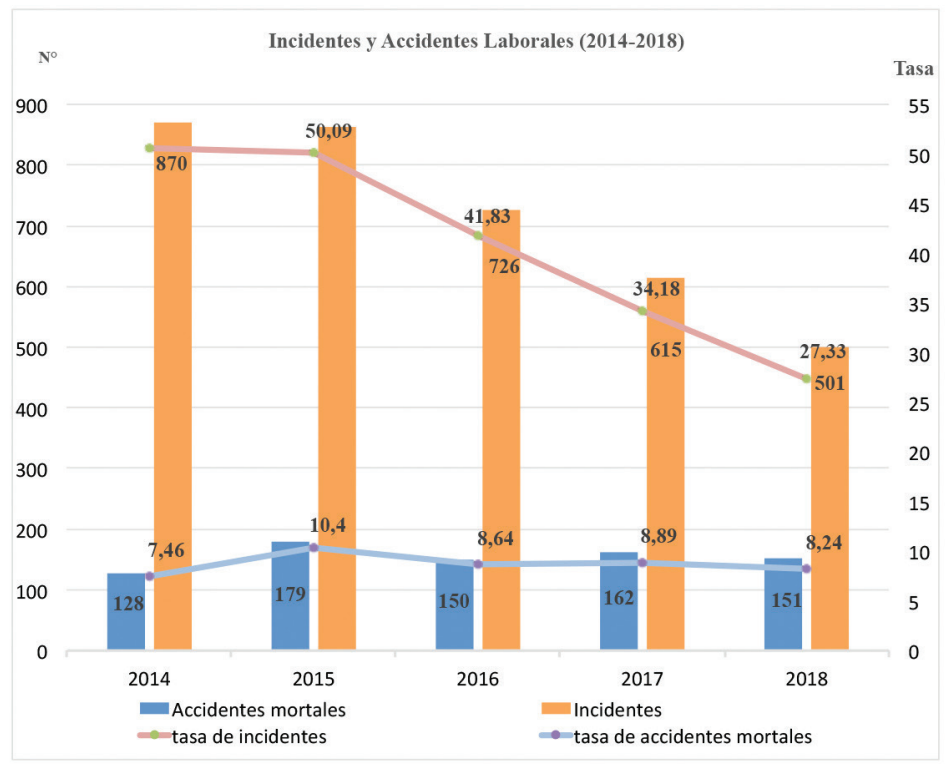

Fuente: Elaboración propia (datos del MTPE).

\section{Técnica observacional}

En el caso de las galerías, se visitó la galería "Mesa Redonda" y otras ubicadas en Lima (todas alojan a microempresarios comerciales en su mayoría), se ha considerado a estas galerías en el estudio como una empresa, por ser una instancia de libre circulación para observar sin obstáculos las actitudes sobre seguridad y salud en el trabajo, tal como que utilizan contenedores de metal como áreas de trabajo y almacenes al igual que en la zona comercial de "Las Malvinas" (donde hubo un incendio con dos muertos y varios heridos). Se observó la faltan de extintores y escasas y no adecuadas señalizaciones; asimismo, en los pasadizos por donde se desplaza el público se encontraron bienes que están a la venta u otros enseres, e inclusive, se observó conexiones eléctricas sueltas, que son situaciones anómalas proclives a riesgos.

También se visualizó que, en forma por demás incongruente con la lógica, cuelgan objetos o regalos a la venta hechos con materiales inflamables como muñecas de plástico, peluches de tela, muy cerca de las cajas de energía eléctrica, propiciando desde luego riesgos potenciales.

Al comentar sobre este grupo de unidades empresariales se ve que prima la informalidad operativa en materia de seguridad del trabajo, pues tendrán toda su documentación en regla, es decir, son formales como negocio o empresa, pero no cumplen con las normas de seguridad y otras, por lo que se le podría llamar que actúan en 
una situación de informalidad operativa, produciéndose por ello diversos accidentes, lo que permite inducir que el comportamiento de este grupo no es el más adecuado, incluso se puede afirmar que, en lugar de no prevenir los riesgos, los generan.

\section{Técnica documental}

Se presentan para el mismo objetivo cuatro casos con sentencia de grandes empresas que llegaron hasta la figura procesal judicial de casación, es decir, el grado más alto de deslinde judicial en el sistema legal peruano, y que se simplifican en la tabla 4, expresando que las empresas evaluadas representan a diversos lugares del país e igualmente las normas infringidas corresponde a la aplicación de la Ley de Seguridad y Salud en el Trabajo

Tabla 4. Casos judicializados con sentencia en contra de empresas por indemnización de daños y perjuicios en accidentes mortales

\begin{tabular}{|c|c|c|c|c|}
\hline $\begin{array}{c}\text { No. Exp. Casación } \\
\text { y año }\end{array}$ & Empresa & Extractos de la sumilla & Norma infringida & Materia \\
\hline $11947-2015$ & $\begin{array}{l}\text { V-Sur Empresa } \\
\text { Vigilancia } \\
\text { Empresa Río } \\
\text { Branco }\end{array}$ & $\begin{array}{c}\text { La prevención es una } \\
\text { obligación laboral del } \\
\text { empleador, lo cual incluye ... } \\
\text { actividades de capacitación } \\
\text { u otra índole en el ejercicio } \\
\text { de la actividad subordinante } \\
\text { que no ha realizado } \\
\text { convenientemente el } \\
\text { empleador }\end{array}$ & $\begin{array}{l}\text { T.P. Principio de } \\
\text { Prevención }\end{array}$ & $\begin{array}{c}\text { Indemnización } \\
\text { por daños y } \\
\text { perjuicios } \\
\text { (accidente) }\end{array}$ \\
\hline $1225-2015$ & $\begin{array}{l}\text { Constructores } \\
\text { Interamericano }\end{array}$ & $\begin{array}{c}\text { Incumplimiento del } \\
\text { empleador de normas } \\
\text { de seguridad y salud } \\
\text { en el trabajo (conducta } \\
\text { injustificada) }\end{array}$ & Varias normas & $\begin{array}{c}\text { Indemnización } \\
\text { por daños y } \\
\text { perjuicios } \\
\text { (accidente) }\end{array}$ \\
\hline $4258-2016$ & Transporte CIVA & $\begin{array}{c}\text { El daño sufrido por el } \\
\text { trabajador se atribuye al } \\
\text { incumplimiento por el } \\
\text { empleador de un deber de } \\
\text { prevención }\end{array}$ & $\begin{array}{l}\text { Deber de } \\
\text { prevención } \\
\text { artículo } 53\end{array}$ & $\begin{array}{c}\text { Indemnización } \\
\text { por daños y } \\
\text { perjuicios } \\
\text { (Accidente) }\end{array}$ \\
\hline $5741-2017$ & $\begin{array}{l}\text { Yanacocha } \\
\text { S.R.L. }\end{array}$ & $\begin{array}{c}\text { Acreditado accidente e } \\
\text { invalidez. No adopción } \\
\text { medidas para salvaguardar } \\
\text { la Seguridad y Salud en el } \\
\text { trabajo }\end{array}$ & Varias normas & $\begin{array}{c}\text { Indemnización } \\
\text { por daños y } \\
\text { perjuicios } \\
\text { (accidente) }\end{array}$ \\
\hline
\end{tabular}

Fuente: Elaboración propia (Casaciones del Poder Judicial Peruano)

Básicamente todos estos accidentes se refieren al incumplimiento del empleador sobre el deber de prevención; un caso por no dar capacitación suficiente, dado el trabajo riesgoso, los otros casos por incumplimiento de las normas sin haber justificación alguna, y cuyo resultado tuvo como consecuencia accidentes mortales; 
refiriendo que estas empresas son de mayor solvencia económica o denominadas grandes, pues incluyen, inversión extranjera.

Con esta técnica y para los mismos fines se evaluó y analizó otro grupo de empresas de diferentes rubros que tuvieron accidentes laborales y que fueron publicados en los diarios El Comercio y El Popular, acontecidos en Lima en diversos años en el periodo de estudio que refiere ciertos comportamientos empresariales (tabla 5).

Tabla 5. Muestra de reportes periodísticos sobre accidentes de trabajo 2014-2017

\begin{tabular}{|c|c|c|c|}
\hline Fecha publicación-Fuente & Empresa y sector & Ocurrencia - Daño & Causa - comentarios \\
\hline $\begin{array}{l}\text { 13-jul-2017 } \\
\text { Diario El Popular }\end{array}$ & $\begin{array}{l}\text { Zeta Gas - Unidad } \\
\text { Transporte de gas } \\
\text {-minería y gas }\end{array}$ & $\begin{array}{c}\text { Explosión-Daños } \\
\text { materiales (carrocería de } \\
\text { seis vehículos y otros) y } \\
\text { humanos (12 personas } \\
\text { heridas entre graves y } \\
\text { leves) }\end{array}$ & $\begin{array}{c}\text { No revisión técnica de balones } \\
\text { de gas }\end{array}$ \\
\hline $\begin{array}{l}\text { 13-jun-2017 } \\
\text { Diario El Popular }\end{array}$ & $\begin{array}{l}\text { Almacén Galería } \\
\text { Mesa Redonda }\end{array}$ & $\begin{array}{l}\text { Incendio - Daños } \\
\text { materiales ( } 26 \text { puestos } \\
\text { quemados) - Humanos } \\
\text { (herido el vigilante). }\end{array}$ & $\begin{array}{l}\text { Negligencia en las medidas } \\
\text { de seguridad (tercera vez que } \\
\text { sucede en los últimos } 5 \text { años) }\end{array}$ \\
\hline $\begin{array}{l}\text { 03-mar-2016 Diario El } \\
\text { Popular }\end{array}$ & $\begin{array}{l}\text { Empresa Grifo- } \\
\text { Cantalao - Repsol } \\
\text { - minería y gas }\end{array}$ & $\begin{array}{c}\text { Explosión - Daños } \\
\text { materiales (ventanas } \\
\text { rotas, etc.) - Humanos } \\
\text { (trabajadora grifo muerta - } \\
\text { dos heridos) }\end{array}$ & $\begin{array}{l}\text { Hipótesis policial: Fuga de gas } \\
\text { por condiciones no adecuadas } \\
\text { o una mala manipulación }\end{array}$ \\
\hline $\begin{array}{l}\text { 16-feb-2016 Diario El } \\
\text { Popular }\end{array}$ & $\begin{array}{l}\text { Concesionaria - } \\
\text { Línea } 3 \text { del metro } \\
\text { Lima - Sector } \\
\text { construcción }\end{array}$ & $\begin{array}{l}\text { Excavación - exploración } \\
\text { - Daño material y humano } \\
\text { (muerte de trabajador por } \\
\text { asfixia) }\end{array}$ & $\begin{array}{l}\text { Hipótesis: Sin implementos } \\
\text { adecuados para oxigenarse. }\end{array}$ \\
\hline $\begin{array}{c}\text { 24-ene-2015 } \\
\text { Diario El Popular }\end{array}$ & $\begin{array}{l}\text { Fábrica de Pinturas } \\
\text { Industria }\end{array}$ & $\begin{array}{l}\text { Incendio - Daño material } \\
\text { (fabrica en destrucción, } \\
\text { activos fijos) y humanos } \\
\text { (contaminación química } \\
\text { gases población) }\end{array}$ & $\begin{array}{l}\text { Ubicación en zona urbana } \\
\text { y no industrial (Licencia } \\
\text { indebida). Descuido en el } \\
\text { almacenamiento de productos } \\
\text { químicos inflamables como } \\
\text { éter, bencina, preservantes, } \\
\text { alcohol y otros }\end{array}$ \\
\hline $\begin{array}{l}\text { 11-ene-2014 } \\
\text { Diario El Comercio }\end{array}$ & $\begin{array}{c}\text { Restaurante Hotel El } \\
\text { Dorado }\end{array}$ & $\begin{array}{c}\text { Incendio - Daño material } \\
\text { (infraestructura del local) - } \\
\text { Daño Humano }\end{array}$ & $\begin{array}{l}\text { Mala maniobra en la cocina. } \\
\text { El establecimiento no cumplía } \\
\text { con la revisión técnica de } \\
\text { acuerda con la normativa de } \\
\text { defensa civil }\end{array}$ \\
\hline
\end{tabular}

Fuente: Elaboración propia.

Como se aprecia en este grupo de empresas, las causas e indicios de las ocurrencias muestran que el empresario en algunos casos tiende a trabajar sin cumplir con las normas y reglamentos legales, porque considera que es pérdida de tiempo (Gestión, 2018); y también hay condescendencia de los municipios en otorgar licencias fundamentadas en razones que no son racionales o técnicas ni concordantes con la ley (informalidad legal). De otro lado, las inspecciones de Defensa Civil encuentran negocios que no cumplen con los implementos de seguridad, dispuestos por la norma 
técnica o igualmente no cumplen con la infraestructura señalada por la norma de seguridad; en ambos casos se generan riesgos potenciales que se pueden evitar. En otras situaciones, las empresas entregan implementos o herramientas desgastadas o deterioradas, generando riesgos en lugar de evitarlos.

\section{Encuestas}

Con la técnica de encuesta se evalúo a 30 trabajadores de cuatro empresas: Insumex SA, Empresa Agregados Calcáreos SAC, Molitalia SA y Donofrio (todas localizadas en Lima). Se hicieron preguntas cerradas, y se obtubieron los siguientes resultados (tabla 6).

(Después de la pregunta incluimos casillas de respuestas el "\% sí" afirmativa y el "\% no" negativa, en este último incluimos los que no contestan, dudan o expresan otra cosa, ya que el interés es que la empresa se preocupe en la seguridad del trabajador y no otra cosa, la tercera casilla es el \% de la respuesta "sí', que debe tender al $100 \%$, esto ocurre cuando a una empresa le interesa plenamente la seguridad y salud del trabajador).

Tabla 6. Resultado de encuesta en relación con cinco preguntas sobre seguridad en el trabajo

\begin{tabular}{cccccc}
\hline Pregunta & $\%$ sí & $\%$ no & $\%$ del sí & Comentarios \\
\hline $\begin{array}{c}\text { ¿Usted recibe capacitación } \\
\text { durante el año? }\end{array}$ & 19 & 11 & 60 & $\begin{array}{c}\text { Rubro importante, todo el personal deba tener } \\
\text { capacitación, algo falla en estas empresas. }\end{array}$ \\
\hline $\begin{array}{c}\text { ¿Sus herramientas se encuentran } \\
\text { en un buen estado? }\end{array}$ & 22 & 8 & 67 & $\begin{array}{c}\text { Las herramientas no están en una condición } \\
\text { técnica adecuada; se tiene que mejorar en este } \\
\text { rubro. }\end{array}$ \\
\hline $\begin{array}{c}\text { ¿La empresa le brinda los } \\
\text { implementos de seguridad } \\
\text { correspondiente? }\end{array}$ & 26 & 4 & 85 & $\begin{array}{c}\text { En este aspecto las empresas están más } \\
\text { fortalecidas, empero el trabajador muchas veces } \\
\text { no usa los implementos, llegando al extremo de } \\
\text { decir que son un estorbo. }\end{array}$ \\
\hline $\begin{array}{c}\text { ¿Las medidas de seguridad se } \\
\text { encuentran bien implementadas } \\
\text { en caso de un evento dañoso } \\
\text { (explosión, terremoto)? }\end{array}$ & 15 & 15 & 50 & $\begin{array}{c}\text { La mitad de los trabajadores considera que no se } \\
\text { encuentran bien implementadas o no satisfacen } \\
\text { ciertas condiciones ante un evento de peligro. }\end{array}$ \\
\hline
\end{tabular}

Fuente: Elaboración propia (Encuesta realizada en diciembre del 2014 por un grupo de alumnos de la UNMSM supervisada por el autor).

Se puede concluir que existe alguna preocupación en este grupo empresarial, pero no es la más idónea, porque hay deficiencias en la entrega de equipos, en la implementación de un protocolo de evacuación en casos de accidentes. Se quiere que las respuestas reflejen un porcentaje cercano al 100\%, siendo por supuesto una prevención ideal al 100\%, o, “cero" riesgos, emulando a los denominados círculos de calidad, cuando se persigue el "cero" defectos; pero en seguridad del trabajo siempre hay una probabilidad de ocurrencia, entonces se debe apostar por un nivel de accidentes mínimo o el menor posible. 


\section{Entrevistas}

Igualmente, se presentan entrevistas de expertos en seguridad del trabajo.

Tabla 7. Entrevistas a expertos en seguridad y salud en el trabajo

\begin{tabular}{ccc}
\hline Entrevistado/a & Extractos de entrevista & Comentarios \\
\hline $\begin{array}{c}\text { Joseph Zumaeta Gerente de } \\
\text { Aptitud } \\
\text { (Bussines Empresarial, } \\
\text { 2018) }\end{array}$ & $\begin{array}{c}\text { "Las empresas deben crear un ambiente } \\
\text { seguro y desarrollar una cultura de } \\
\text { autocuidado, de salud, seguridad y } \\
\text { prevención de riesgos laborales". }\end{array}$ & $\begin{array}{c}\text { Se enfoca en la prevención tanto en la } \\
\text { empresa como en el trabajador, dado } \\
\text { que la seguridad del trabajador es un } \\
\text { sistema que es problema de todos. }\end{array}$ \\
\hline "Han pasado 10 años del DS No. \\
009-2005-TR donde se establecen \\
estándares mínimos de proteción \\
para las actividades económicas, sean \\
o no de alto riesgo... estamos lejos de \\
alcanzar un compromiso con la cultura \\
de prevención de riesgos".
\end{tabular}

Fuente: Elaboración propia.

Las entrevistas refieren la falta de compromiso hacia una cultura de prevención de riesgos tanto de los empleadores (deber de prevención), así como del trabajador (eluden su obligación de protegerse o el autocuidado), rescatando un comportamiento negativo en ambos lados (reiteramos que al evaluar a los trabajadores se evalúa el comportamiento de la empresas donde trabajan); resaltando la respuesta de uno de los entrevistados: "si no se alcanzan mayores tasas de formalidad empresarial y productiva, las mejoras en materia de seguridad, salud y trabajo o la disminución de riesgos de accidentes, es una ilusión".

Por todo ello, se halló que hay sesgos débiles de la seguridad en el trabajo empresarial, en consecuencia, las empresas deberán actuar con mayor compromiso, apuntando a disminuir o eliminar los riesgos laborales, dado que además de ser una responsabilidad legal, es una actitud moral con el prójimo, inherente al respeto a la vida o integridad física y psíquica. 
De otro lado, es juicioso aclarar que en este tipo de accidentes existe una relación: empresa-trabajador, donde el acreedor es el trabajador y el empleador es el deudor. De tal manera que ocurrido un accidente acontecerá una sucesión de costos y gastos que la empresa debe afrontar con la consiguiente afección de la rentabilidad en el periodo de la ocurrencia.

Diversos autores tratan de clasificar o simplificar estos costos y gastos; así, algunos lo dividen en dos partes: costos directos-visibles-asegurables que son los desembolsos que hace la empresa en beneficio directo del accidentado, dentro ellos se considera el pago de compensación salarial, gastos de asistencia médica, indemnizaciones y sepelios. Y los costos indirectos-ocultos-no asegurados que son aquello desembolsos que son pérdidas que sufre una empresa como consecuencia indirecta de los accidentes.

Asimismo, se incluyen costos que podría incidir en uno o varios de estos componentes referidos al sistema productivo cuya evaluación varía de acuerdo con el impacto del suceso, a saber:

- Mano de obra: Referido al personal del sistema productivo que se ve impactado.

- Maquinaria: Equipos que se deterioren o se destruyan además de interrumpir la producción.

- Materiales: Son la materia prima o insumo, artículos semielaborados o ya productos que estaban acabados.

- Instalaciones y activos fijos: Es la edificación ocupada y las instalaciones eléctricas, caloríficas, equipos de cómputo u otros de las oficinas.

- Tiempo: Es una correlación del accidente o evento que paraliza el sistema productivo parcial o total, diferente a los puestos cercanos del trabador lesionado.

Todo este enjambre de costos es evaluado contablemente y algunos pueden estar asegurados, pero otros no. Bird, (1993) indica en forma proporcional, que si: un dólar es el costo por lesión y enfermedad (costos médicos, costos de compensación, costos asegurados), de 5 a 50 son gastos contabilizados por daños a la propiedad (costos sin asegurar), y de 1 a 3 (costos misceláneos sin asegurar). Es decir, los costos y gastos que aparentemente no se ven, son mucho más que los visibles o apreciables.

Como se observa, los costos son altos para dejar la seguridad y salud en el trabajo, sin una adecuada prevención, aspecto que impulsa a la empresa a buscar el mejor método o condiciones idóneas donde se desarrolle el trabajo, que se traduce en un ambiente laboral seguro. En caso contrario, cuando se obvia esta preocupación, lo más seguro es la ocurrencia de accidentes.

Como se deduce, un accidente laboral trae resultados que se traducen en costos que tiene que asumir la empresa en diversos rubros, ahora bien, si la empresa desea 
disminuir estos costos, deberá tomar las medidas de prevención que la ley y la lógica empresarial lo exigen. Para ello existen estudios muy amplios que evalúan las políticas empresariales que incluyen programas de prevención de acuerdo con el tipo de empresa, que deben ser abordadas convenientemente (Alarcón y Maguiña, 2018).

Ahora bien, pensando en la dualidad trabajador-empresa, cuando en la empresa prima la prevención de riesgos inherente a una gestión integral, además de sun buen clima laboral, es más probable que el trabajador mejore su rendimiento, pues, asume que la empresa lo valora, lo cuida; en consecuencia, lo lleva a adoptar mejores actitudes laborales, tal como el desarrollo de ideas innovadoras, y mejoras en provecho de la productividad empresarial, entre otras.

Desde este ángulo, son muchos los estudios que persisten en que la mejor opción para optimizar la seguridad y salud en el trabajo en una empresa es integrarlo a la gestión estratégica de la empresa (Vega, 2017). En la actualidad ello se fundamenta en el ISO 45001, de reciente aparición, que remplazará en los próximos años a la OHSAS 18001.

Finalmente, se expresa que las empresas necesitan de los trabajadores y los trabajadores necesitan de las empresas, esto es una interacción básica, en el entendido de que cuando hay accidentes es probable que algo está fallando en materia de gestión de la seguridad, que conlleva deducir que no se le ha dado la importancia debida a esta materia, por lo tanto es un compromiso del binomio empresario-trabajador retroalimentar la gestión de seguridad en el trabajo. Desde esta perspectiva, igualmente es ineludible la atención del Estado en la identificación y evaluación de las condiciones de trabajo y exposiciones (Cano y Francia, 2018), el que a través de sus políticas y estrategias debe asumir un rol, más que de fiscalizador, de sensibilizador empresarial.

Concluyendo que es un deber del mismo trabajador cuidar de su salud y de su vida, así como la de sus compañeros trabajadores, y desde el punto de vista del empresariado, sentir que el trabajador es un ser humano valioso tanto para su familia como para la empresa e incluso para la sociedad. Más aún cuando creemos que la seguridad en el trabajo en tiempos modernos de responsabilidad social, sostenibilidad empresarial y humanización del trabajo cumple un rol preponderante en todo sistema económico empresarial.

\section{Conclusiones}

Las empresas deben internalizar en sus cuadros directivos que asumir la seguridad en el trabajo significa resguardar un derecho humano fundamental de la persona en su calidad de trabajador, al mismo tiempo que se protege la economía empresarial. Y más que meras obligaciones de cumplimiento, las empresas deben sopesar con 
satisfacción que resguardar la vida de su trabajador permite ganar en productividad y competitividad ante el mercado y la sociedad.

En tal sentido, se encuentran debilidades en el comportamiento empresarial, sean estas micro, pequeñas, medianas o grandes empresas, soslayan un relativo interés en el resguardo de la seguridad del trabajador, revertir esta actitud, sosteniendo y asumiendo que un accidente se puede prevenir para evitar gastos indeseados, gestará comportamientos responsables y ceñidos a un mejor rendimiento empresarial, que es concordante con la formalidad y el respeto a la ley en todo sus aspectos, diferenciándose de las empresas informales que nada o poco hacen por la prevención de accidentes, adoptando un comportamiento ajeno a la dignidad del trabajador y la vida humana.

Es importante que el empresariado como parte de su política de desarrollo humano invierta en un sistema de seguridad en el trabajo idóneo, desterrando la concepción errada que es un gasto, tal como se presume en algunos casos como cuando las micros o pequeñas empresas prefieren mantener una herramienta o equipo gastado o malogrado para el trabajo, sin tener en cuenta que mayor será el gasto si no lo hacen.

La autoridad pública debe tener legislaciones y reglamentos claros para mejor cumplimiento, control y fiscalización de las unidades empresariales e institucionales, así como tener funcionarios y o empleados idóneos y honrados al hacer inspecciones y otorgar licencias, cumpliendo solo con el orden técnico y racional; aspectos que en las fiscalizaciones e inspecciones de algunos negocios o empresas no ha tenido en cuenta. Igualmente le compete cumplir con un rol sensibilizador, tanto con el trabajador como también con la empresa.

Además, los resultados evidencian que las empresas tendrán que mejorar su rendimiento en materia de seguridad en el trabajo, más aún cuando a partir de marzo del 2018 se ha implantado a nivel internacional el uso del ISO 18001 para integrar la gestión de la seguridad en el trabajo en la gestión estratégica de la empresa.

En general todo cambio para mejorar debe ser una práctica ineludible en toda empresa que se precie moderna y sostenible, adoptando los últimos sistemas, procedimientos o herramientas del mundo tecnológico, no obstante, conforme a lo analizado y evaluado, no solo es problema de tecnología si no depende de la actitud del ser humano, sea empresario o trabajador.

Como corolario final de ese estudio se expresa que se vive en un mundo de riesgos, las empresas -se evidencia que en su gran mayoría las micros y pequeñas lo omiten-, deben en lo posible utilizar sistemas, técnicas e instrumentos para administrar la incertidumbre de un evento riesgoso, en el sentido de que un accidente sea prevenido y mitigado, evitando ocurrencias indeseadas y sus consecuencias. 


\section{Referencias}

Agencia Andina de Noticias. (2019). Reporte del Instituto Nacional de Estadística del Perú. Recuperado el 22 de setiembre 2019, de https:/andina.pe/agencia/noticiaccl-cinco-regiones-concentran-45-del-pbi-sin-contar-lima-740442.aspx

Alarcón, S. N., y Maguiña, K. A. (2018). Implementación de un sistema de gestión de seguridad y salud ocupacional para disminuir los riesgos laborales en el Centro Médico Villa. (Tesis pregrado). Universidad Cesar Vallejo, Huaraz. Recuperado el 10 de diciembre del 2019, de http://repositorio.ucv.edu.pe/handle/ $\mathrm{UCV} / 26121$ ?show $=$ full

Bird, F. (1993). Administración del control de pérdidas. 1993. Estados Unidos de América.

Business Empresarial. (2018). Entrevista a Joseph Zumaeta, 12 de enero 2018. Recuperado de http: www.businessempresarial.com.pe/

Cano, C., Francia, J. (2018). Estado de avance de la salud de los trabajadores en Perú. Acta Med Perú, 35(1), 3-5.

Céspedes, G. M., y Martínez, J. M. (2016). Un Análisis de la seguridad y salud en el trabajo en el sistema empresarial cubano. Revista Latinoamericana de Derecho Social (22), 3-61.

Chamochumbi, C. (2014). Seguridad e higiene industrial (p. 224). Lima. Fondo Editorial de la Universidad Garcilaso de la Vega.

Diario Gestión. (2016). Perú sale 'jalado’ en sus cifras sobre fatalidad laboral. 30 de junio del 2016. Recuperado el 16 de abril del 2018, de https://gestion.pe/economia/ peru-sale-jalado-cifras-fatalidad-laboral-125466

Escalona, E. (2006). Relación salud trabajo y desarrollo social: visión particular en los trabajadores de la educación. Revista Cubana Salud Pública, 32(1), p. 18.

Fabián, E. R. (2017). Diseño e implementación de sistema de gestión en seguridad y salud ocupacional en la planta de Yauris. (Tesis doctoral). Universidad Nacional del Centro del Perú. Huancayo, Perú. Recuperado el 20 septiembre de 2019, de http://repositorio.uncp.edu.pe/discover

Feo, O. (2010). Crisis global y su impacto sobre el trabajo y la salud. Salud de los Trabajadores, 18(2), 91-94.

Figueroa, N., Ribet, M., Garrido, M., Ramos, M. E., y Enrique, Y. (2013). La gestión de riesgos laborales en las empresas forma parte de su responsabilidad social. Avances, 15(1), 64-75.

Guerrero, E. (2015, enero 24). Ardieron a lo bonzo. El Popular, p. 4. 
Guerrero, E. (2016, marzo 3). Terror por explosión. El Popular, p. 2.

Hernández, C. (2014). Integración de la gestión de seguridad y salud en el trabajo en la responsabilidad social. Estudio de caso en cinco empresas. (Tesis de grado) Universidad Nacional de Colombia - UNAL, Bogotá. Colombia.

Maldonado, Y. (2016, febrero 16). Obrero murió asfixiado dentro de pozo en Panamericana Norte. El Popular, p. 2.

Maldonado, Y. (2017, junio 13). ¡Infierno! El Popular, p. 2.

Mejía, C., Cárdenas, M., y Gomero, R. (2015). Notificaciones de accidentes y enfermedades laborales al Ministerio de Trabajo. Perú 2010-2014. Revista Peruana de Medicina Experimental y Salud Publica, 32, 526-531.

Ministerio de Trabajo y Promoción del Empleo. (2019). Boletín Estadístico. Notificaciones de accidentes de trabajo, incidentes peligrosos y enfermedades ocupacionales. Lima. Recuperado el 20 de diciembre 2018, de https://www. gob.pe/institucion/mtpe/informes-publicaciones/283908-boletin-estadisticonotificaciones-de-accidentes-de-trabajo-incidentes-peligrosos-y-enfermedadesocupacionales

Olavarri, R. (2014). Economía de la prevención: costes y beneficios de la prevención de la empresa. En Salud Laboral. Conceptos y técnicas para la prevención de riesgos laborales. España: Elsevier.

Organización Internacional de Trabajo - OIT. (2019). Seguridad y salud en el centro del futuro del trabajo. Aprovechar 100 años de trabajo. Lima. Recuperado el 10 de diciembre de 2019, de https://www.ilo.org/safework/events/safeday/ WCMS_686762/lang--es/index.htm.

Ortega, J. A., Rodríguez, J. R., y Hernández, H. (2017). Importancia de la seguridad de los trabajadores en el cumplimiento de procesos, procedimientos y funciones. Revista Academia \& Derecho, 8(14), 155-176.

Palacios, M. E., Tamez, S., y González, R. (2014). La salud de los trabajadores y su determinación social. En Esteban, J. M., Palacio, M. E., Paz, M. P., García, G. S. y Moreno, L. Salud, ambiente y Trabajo (pp. 171-189). México: McGraw-Hill Education.

Pinilla, J. (2019). Perspectiva de la seguridad y salud en el trabajo InterempresasProtección Laboral. Recuperado el 10 de diciembre de 2019, de https://www. interempresas.net/Proteccion-laboral/Articulos/252376-Perspectivas-de-laseguridad-y-la-salud-en-el-trabajo.html

Poder Judicial. (2015). CAS. No. 11947-2015 PIURA. Publicado en el Diario Oficial El Peruano el 2 de mayo 2016.

Poder Judicial. (2017). CAS. No. 10398-2017 LIMA. 
Poder Judicial. (2016). CAS. No. 4258-2016 LIMA. Publicado en Diario Oficial El Peruano el 30 de enero del 2017.

Poder Judicial. (2016). CAS. No. 1225-2015 LIMA. Sentencia del 07 de abril del 2017.

Porter, P. (2009). Ser competitivo. (9a. ed.). Chile: Deusto.

Raffo, E. (2016). Introducción a la seguridad y salud en el trabajo (p. 287). Lima Impresión Editorial. Colecciones Jovic.

Riaño, M. I., y Palencia, F. (2016). Dimensión económica de la seguridad y la salud en el trabajo: una revisión de la literatura. Revista Gerencia. Política. Salud, 15(30), 24-37.

Roble, J. (2017, julio 13). Pánico por explosión. El Popular, p. 2.

Salguero, F. M. (2017). Análisis y evaluación de la investigación de accidentes laborales como técnica preventiva en España. (Tesis doctoral). Universidad de Málaga. Málaga, España.

Silva, J. (2001). La expansión del derecho penal. Aspectos de la política criminal en las sociedades posindustriales. Revista de Derecho Penal (2), 293-297

Suasnavas, P. R., Andrade, A. R., Granda, K. M., Dávalos, H. P., Cárdenas, H. P., y Gómez, A. R. (2019). Responsabilidad social y gestión de la seguridad y salud en el trabajo: panorama actual de las empresas ecuatorianas. Revista Espacios, 40(04). Recuperado el 22 de setiembre de 2019, de https://www.researchgate. net/publication/331043859_Responsabilidad_social_y_gestion_de_la_ seguridad_y_salud_en_el_trabajo

Tagle, N. M., y del Carmen, R. M. (2019). Efectividad de las capacitaciones en prevención de seguridad y salud ocupacional en trabajadores. (Tesis de grado) Universidad Norbert Wiener. Lima, Perú.

Ugaz, M., y Soltau, S. (2011). Implicancias de la Ley de Seguridad y Salud en el Trabajo. Derecho \& Sociedad (37), 166-180.

Universidad Internacional de Valencia. (2016, mayo). La siniestralidad laboral en Europa y Latinoamérica: Una visión comparada. Recuperado el 16 de abril 2017, de https://www.aepsal.com/wp-content/uploads/2016/05/Siniestralidad-laboralen-Europa-y-Latinoamerica.pdf.

Vega, N. C. (2017). Nivel de implementación del Programa de Seguridad y Salud en el Trabajo en empresas de Colombia en territorio antioqueño. Cuadernos de Salud Pública, 33(6), 166-180. 